\title{
A Novel Three Stage CBIR using Varying Higher-Order Zernike Moments and its Performance Analysis
}

\author{
Dipti Jadhav \\ Assistant Professor \\ Ramrao Adik Institute of \\ Technology
}

\author{
Mohini Patil, Anagha Patil, \\ Lalita Phalak \\ Ramrao Adik Institute of \\ Technology
}

\begin{abstract}
This paper features the novel three stage CBIR system using different orders of Zernike moments. In the first stage, segmentation is done using various image processing techniques. In the second stage, two sets of features each containing 32 Zernike moments and one more set containing 62 Zernike moments with different orders are extracted from the images. This paper also presents an experimental analysis of using different orders of Zernike moments. In the final stage, the experimental results show that the Zernike moments of order 3-10 shows the better results.
\end{abstract}

\section{General Terms}

CBIR.

\section{Keywords}

CBIR, Zernike moments,moments order, analysis, precision, recall etc.

\section{INTRODUCTION}

Content Based Image Retrieval (CBIR) has become a very prominent area in image processing because of its diverse applications in the areas like internet, multimedia, medical image archives, and crime prevention to name a few. The ever increasing demand for image databases has increased the need to store and retrieve digital images. Extraction of visual features, viz., color, texture, and shape is an important component of CBIR. Shape is a primary image feature and is especially useful when the image contains man-made objects [15]. It is also useful for image analysis, object identification and image filtering applications. In image retrieval, it is important for some applications in which shape representation is invariant under translation, rotation, and scaling. Therefore, we will focus on shape representations that are transformation invariant. Region-based shape descriptors can be applied to more general shapes. However, contour-based shape descriptors have limitations of extracting complex shapes. Hence, region based shape descriptors viz., Moment Invariants (MI), Zernike Moments (ZM), and Legendre Moments (LM) are preferred to represent the shape content of an image [15].

\section{LITERATURE SURVEY}

CBIR also known as Query By Image Content (QBIC) is the application of computer vision techniques to the image retrieval problem. The term 'content' in this context refers to colors, shapes, textures, or any other information that can be derived from the image itself [1]. Many scientists have proposed many techniques in order to retrieve the information or data present in the image. The systems Walrus and Wlindsurf both uses region features extracted from wavelet transform while Walrus uses Birsch segmentation to obtain regions [2]. In [2] Abhinav Deshpande and et al provides a CBIR technique that uses both Zernike moment descriptor and Gabor feature. In [3] Basavaraj S Anami et al. have outlined the efficiency analysis of some of the boundary based and contour based shape feature extraction techniques. In [3] the Zernike moments are calculated upto the fourth order moments. Dipti Jadhav et al[4] have proposed a hybrid technique using high order colour moments(skew and kurtosis)and texture(CCM and GLCM) for effective retrieval. A fast and accurate approach to the computation of Zernike moments is presented in [5]. This approach computes the Zernike basis function with the fixed exact co-efficient which aims at reducing the computation efforts or time. Manimala Singha and K. Hemachandran[6] have proposed a CBIR technique using Wavelet Based Color Histogram Image Retrieval. Shriram K V et al.[7] gives an analysis of the CBIR system using Colour and Texture. The author also suggests using Entropy as a feature alongwith Region of Interest (ROI) to help the user select the region from the image which can be used for training. Dengshang et al.[8] discusses various shape representation and description techniques comparing region based and contour based techniques. Tohid Shedghi [9] has proposed a technique with texture using Gabor filter as a primary feature and shape as secondary feature.

The few research efforts discussed above provides the basis of the CBIR using either colour, texture or both for retrieval while the few have used shape. An image is a good composition of colour, texture and shape. Shape is a useful visual component of an image. An efficient retrieval requires that the shape descriptor retrieves images from the database even if the images have undergone rotation, translation, scaling or are affinely transformed. This paper proposes a novel three stage CBIR technique which uses image segmentation, moments (Zernike moments) and shape matching with good precision and recall. This paper also performs an analysis of 3 different sets of moments order.

\section{PROPOSED METHODOLOGY}

The block diagram of the proposed method is given below in Fig 1. The method described in this work contains three major stages-i) image segmentation ii) content retrieval using moments and then iii) shape matching. 


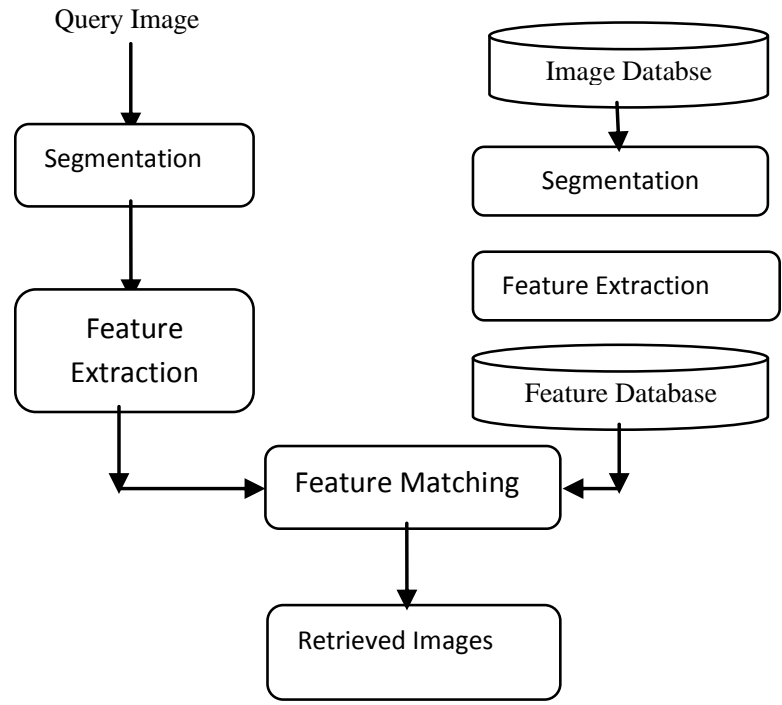

Fig.1. Block diagram of the proposed system.

The objective of the image segmentation stage is to extract the shapes / regions from the image. Once the image is segmented and regions are obtained, features belonging to the obtained regions are recorded. The next step is to use appropriate descriptors for the obtained regions. The descriptors are used for matching regions of different images. Regional descriptors i.e., moment invariants describe the content or the interior of the region [8]. The shape is represented with a feature vector. In the third stage of shape matching, Euclidean distance is used to calculate the distance between the feature vectors of the query image and the image in the database.

\subsection{Segmentation}

The conventional segmentation approaches often fails in segmenting the images with high complexity because of high processing time and different contrast between various parts in the single object. [8] An object can be easily detected in an image if the object has sufficient contrast from the background.

This paper uses edge detection and the basic morphological operations to detect an object in the image.

\subsubsection{Resize the image:}

Zernike Moments are applicable to only $\mathrm{N} * \mathrm{~N}$ matrix, where $\mathrm{N}$ is an even number, we resize each image to $480 * 480$ pixels.

\subsubsection{Detecting edges of object:}

Sobel operator is used to detect the edges. The masks used are $S_{\mathrm{x}}=[-1,-2,-1 ; 0,0,0 ; 1,2,1], \mathrm{S}_{\mathrm{y}}=[-1,0,1 ;-2,0,2 ;-1,0,1]$

\subsubsection{Perform Dilation:}

Dilation causes adding pixels to the boundary of the image. Dilation is done with 2 masks: dilMask $1=[1 ; 1 ; 1]$; dilMask2 $=[1,1,1]$. The weaker edges are made thicker and linked.

\subsubsection{Fill the interior gaps:}

The dilated gradient mask shows the outline of the object, but leaves few holes in the interior of the object. To fill these holes region filling operation is used. This performs flood-fill operations on background pixels of the input image starting from the point specified in the locations. In the proposed work location are holes.

\subsubsection{Remove Connected Objects on Border:}

The object of interest is successfully segmented, but it is not the only object that is found. Any objects that are connected to the border of the image can be removed using the clear border operation.

\subsubsection{Perform Erosion:}

Erosion causes removing pixels from the boundary of the image. In order to make the segmented object look natural, the object is smoothened by eroding the image twice. The mask used is eroMask $=[0,1,0 ; 1,1,1 ; 0,1,0]$.

Finally, a fully segmented image is obtained. The height and width of the desired object is cropped from the rest of the image. The image is resized back to $480 * 480$ pixels. It is the desired region on which Zernike moments are applied. Fig. 2 shows an instance of segmentation of an image from the image database.

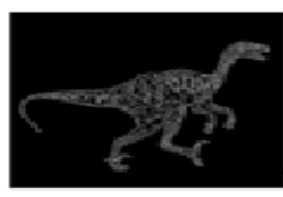

(a)

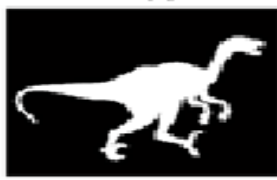

(d)

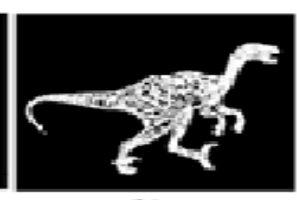

(b)

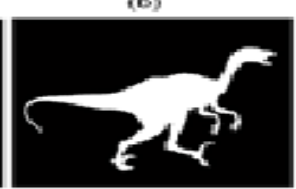

(b)
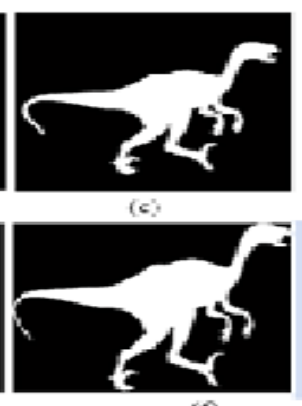

(1)
Fig. 2.(a) Object Edges (b) Dilated Image (c)Region Filling (d) Cleared Border Image (e) Eroded Image (f) Cropped Object.

\subsection{Zernike moments}

Moments are area descriptors and are used to characterize the shape and size of the image. In order to retrieve an image from a large database the descriptor should have enough discriminating power and immunity to noise. In addition, the descriptor should be invariant to scale and rotation, not to mention the computation efficiency. The Zernike moment descriptor has such desirable properties: rotation invariance, robustness to noise, expression efficiency, fast computation and multi-level representation for describing the various shapes of pattern [11]. The complex ZM are derived from Zernike polynomials which are a set of complex orthogonal polynomials, the orthogonality implies no redundancy overlap of information between the moments with different orders and repetitions. This property enables the contribution of each moment to be unique and independent from the information in an image. High-order Zernike moments not only have a high computational complexity, but also represent a high sensitivity to noise. In fact, they may diminish the performance of the system if they are not selected precisely. However, they might be better descriptors of shape and margin characteristics than low-order Zernike moments. The computation of Zernike moments from an input image includes three steps: computation of radial polynomials, computation of Zernike basis functions and computation of 
Zernike moments by projecting the image onto the Zernike basis functions [12]. The complex ZM are derived from Zernike polynomials which are a set of complex orthogonal polynomials defined over the interior of a unit circle $x^{2}+y^{2}=1$. The procedure for obtaining Zernike moments from an input image starts with the computation of Zernike radial polynomials. The real-valued 1-D radial polynomial $R_{n, m}$ is defined as in eq.(1)[13]:

$R_{n, m}(\rho)=\sum_{s=0}^{(n-|m|) / 2}(-1)^{s} \frac{(n-s) !}{s !\left(\frac{(n+|m|)}{2}-s\right) !\left(\frac{n-|m|}{2}-s\right) !} \rho^{n-2 s}$

where $\mathrm{n}$ is a non-negative integer representing the order of the radial polynomial, $\mathrm{m}$ is a positive or negative integer satisfying constraints $n-|m|=$ even and $|m|<=n$ representing the repetition of the azimuthal angle. $\rho$ is the length of vector

from the origin to $(x, y)$ as given in eq. (2)[13]:

$\mathrm{V}_{\mathrm{n}, \mathrm{m}}(\mathrm{x}, \mathrm{y})=\mathrm{V}_{\mathrm{n}, \mathrm{m}}(\rho \sin \theta, \rho \cos \theta)=\mathrm{R}_{\mathrm{nm}}(\rho) \exp (\mathrm{jm} \theta)$.

The complex Zernike polynomials satisfy the orthogonality condition as eq.(3)[13]:

$A_{n, m}=\frac{(n+1)}{\pi} \iint_{x^{2}+y^{2} \leq 1} f(x, y) V_{n m}^{*}(\rho, \theta) d x d y$.

or in polar coordinates as in eq.(4)[13]:

$A_{n, m}=\frac{(n+1)}{\pi} \int_{0}^{2 x} \int_{0}^{1} f(\rho,) R_{\pi m}(\rho) \exp (-j m) \rho d \rho d(4)$

For digital images, the integrals in the above equation can be replaced by summations. Moreover, the coordinates of the image must be normalized into $[0,1]$ by a mapping transform, as shown in Fig.3.

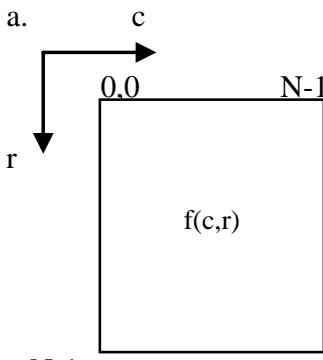

$\mathrm{N}-$

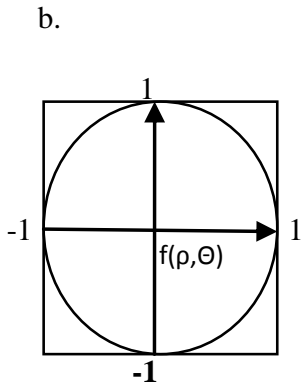

Fig. 3 (a) $\mathbf{N} \mathbf{N}$ digital image with function $\mathbf{f}(c, r)$ and (b) the image mapped onto the unit circle.

The pixels located on the outside of the circle are not involved in the computation of the Zernike moments. Eventually, the discrete form of the Zernike moments for an image with the size $\mathrm{N}^{*} \mathrm{~N}$ is expressed as eq(5) and (6)[12]:

$\mathrm{Z}_{\mathrm{n}, \mathrm{m}}=\frac{(n+1)}{\lambda} \sum_{c=0}^{c=n-1} \sum_{\gamma=0}^{\gamma=n-1} f(c, r) V_{n, m}^{*}(c, r)$

$\mathrm{Z}_{\mathrm{n}, \mathrm{m}}=\frac{(n+1)}{\lambda} \sum_{c=0}^{n-1} \sum_{r=0}^{r=n-1} f(c, r) R_{n, m}\left(\rho_{\mathrm{cr}}\right) e^{-j m \theta \mathrm{cr}}$ where $0 \leq \rho_{\mathrm{cr}} \geq 1$, and $\boldsymbol{\lambda}_{\mathrm{N}}$ is a normalization factor. In the discrete implementation of Zernike moments, the normalization factor must be the number of pixels located in the unit circle by the mapping transform, which corresponds to the area of a unit circle, $\mathrm{p}$, in the continuous domain. The transformed distance, $\rho_{\mathrm{cr}}$, and the phase, $\theta_{\mathrm{cr}}$, at the pixel of (c,r) are given as in eq.(7) and (8). $\mathrm{c}$ and $\mathrm{r}$ denote the column and row number of image, respectively.

$\rho_{\mathrm{cr}=} \frac{\sqrt{(2 c-N+1)^{2}+(2 r-N+1)^{2}}}{N}$

$\theta_{\mathrm{cr}}=\tan ^{-1}\left(\frac{N-1-2 r}{2 c-N+1}\right)$

It can be shown that rotating the object around the Z-axis do not influence the magnitude response of the Zernike moments. Indeed, the rotation only influences the phase response of Zernike moments. In other words as in eq(9):

$\mathrm{Z}_{\mathrm{n}, \mathrm{m}}^{\mathrm{r}}=\mathrm{Z}_{\mathrm{n}, \mathrm{m}} e^{-j \mathrm{j} \omega}\left|\mathrm{Z}_{\mathrm{n}, \mathrm{m}}^{\mathrm{r}}\right|=\left|\mathrm{Z}_{\mathrm{n}, \mathrm{m}}\right|$

where $Z_{n, m}^{r}$ and $Z_{n, m}$ are the moments which are extracted from the object and the rotated object, respectively. The rotation angle is a. The magnitude of the Zernike moment of object and that of rotated object are equal [12].

The Table I given below shows the proposed Zernike moments and its orders.

Table I. The proposed Zernike Moments

\begin{tabular}{|c|c|c|c|}
\hline Group & $\mathbf{n}$ & $\mathbf{m}$ & $\begin{array}{l}\text { Number of } \\
\text { Moments }\end{array}$ \\
\hline \multirow{8}{*}{1} & 3 & 1.3 & \multirow[t]{8}{*}{ 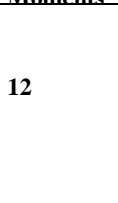 } \\
\hline & 4 & 0.2 .4 & \\
\hline & 5 & 1.3 .5 & \\
\hline & 6 & $0,2,4,6$ & \\
\hline & 7 & $1,3,5.7$ & \\
\hline & 8 & $0,2,4,6,8$ & \\
\hline & 9 & $1,3,5,7,9$ & \\
\hline & 10 & $0,2,4,6,8,10$ & \\
\hline \multirow{8}{*}{2} & 10 & $2,6,10$ & \multirow{8}{*}{32} \\
\hline & 11 & $3,7,11$ & \\
\hline & 12 & $0,4,8,12$ & \\
\hline & 13 & $1,5,9,13$ & \\
\hline & 14 & $2,6,10,14$ & \\
\hline & 15 & $3,7,11,15$ & \\
\hline & 16 & $0,4,8,12,16$ & \\
\hline & 17 & $1,5,9,13,17$ & \\
\hline
\end{tabular}

\subsection{Euclidean distance}

For the input query image, the shape feature of that image is identified and is compared with the images in the database by using Euclidean distance method. By comparing query image with the image database, the distance between images are found and shortest distance is considered as the best match. The images are displayed in the ascending order of the distances. The distance $d$ between the two image feature vectors is calculated by using the following eq (10) [14]:

$\left.\left.\mathrm{d}=\sqrt{\left(\sum_{i=0}^{N}\left(f_{q}\right.\right.}(i)-f_{d}(i)\right)^{2}\right)$

where, $f_{q}(i)$ is the $i^{\text {th }}$ feature vector of the query image, $f_{d}(i)$ is the $i^{\text {th }}$ feature vector of the database image. 


\subsection{Experimental Database:}

The database contains 330 images with 6 categories each containing 55 images for experimental analysis.

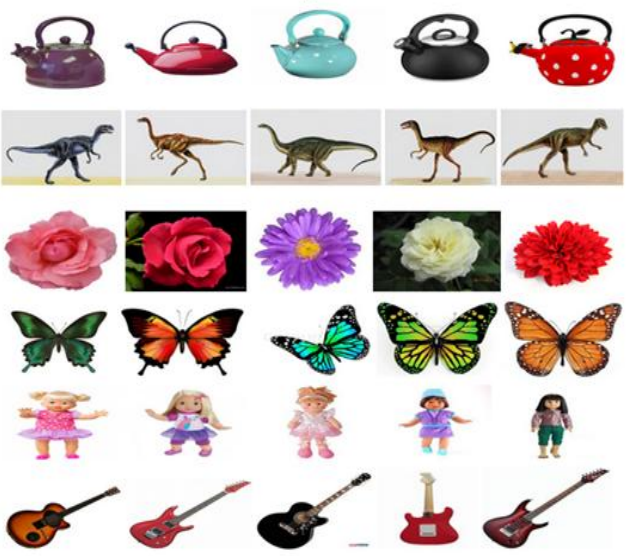

Fig.4. Sample Image Database

The categories include butterflies, teapots, flowers etc. The database is constructed by taking various images from the Internet i.e by creating own database.

\section{Experimental Analysis}

In the proposed system, results are obtained using higher order Zernike moments. Zernike moments up to order 17 are used for extracting shape features. The analysis is done by collecting results for moment orders between 3-10, 10-17 and $3-17$. The results using butterfly and flower as the query image is shown below.

\subsection{Visual Results:}

4.1.1 Results using moments between orders 3-10:

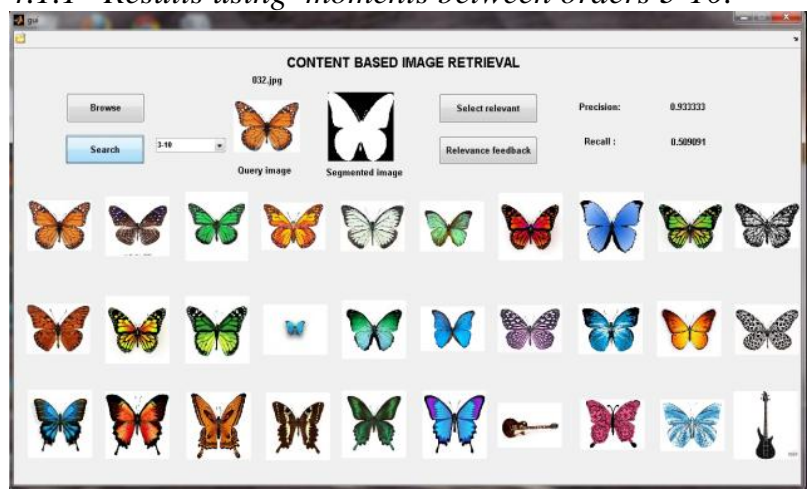

Fig.5. Results of butterfly as a query image.

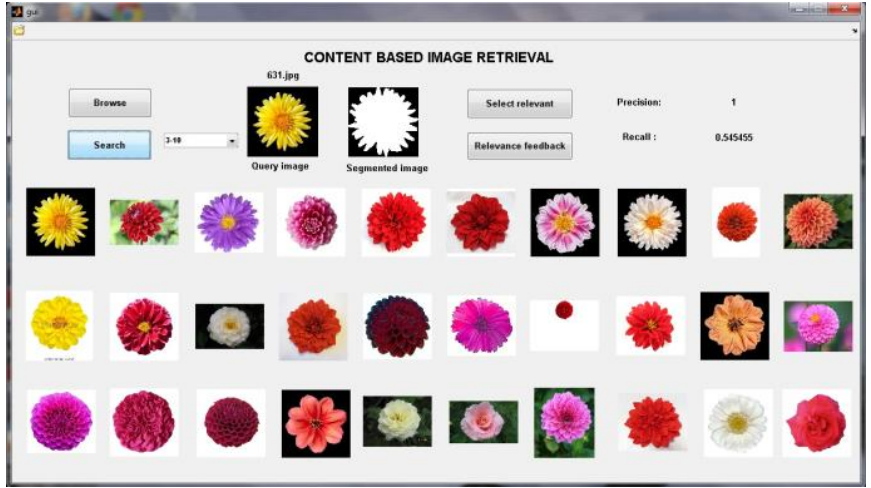

Fig.6. Results of flower as a query image.

4.1.2 Results using moments between orders 10-17:

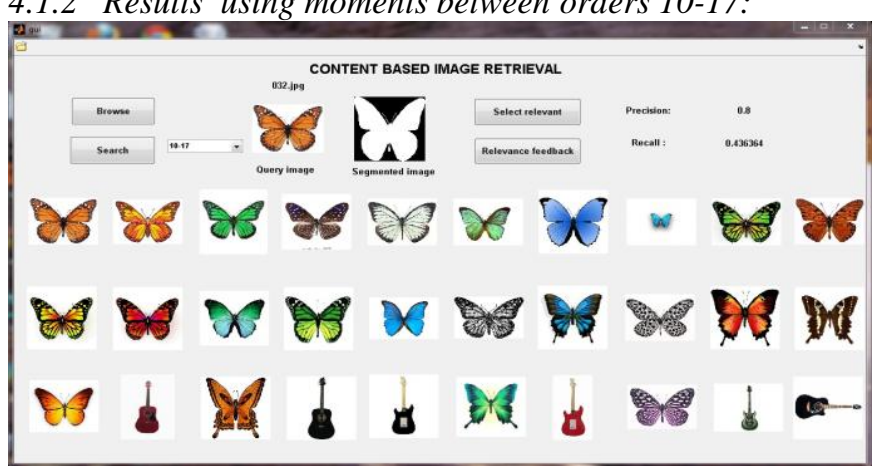

Fig.7. Results of butterfly as a query image.

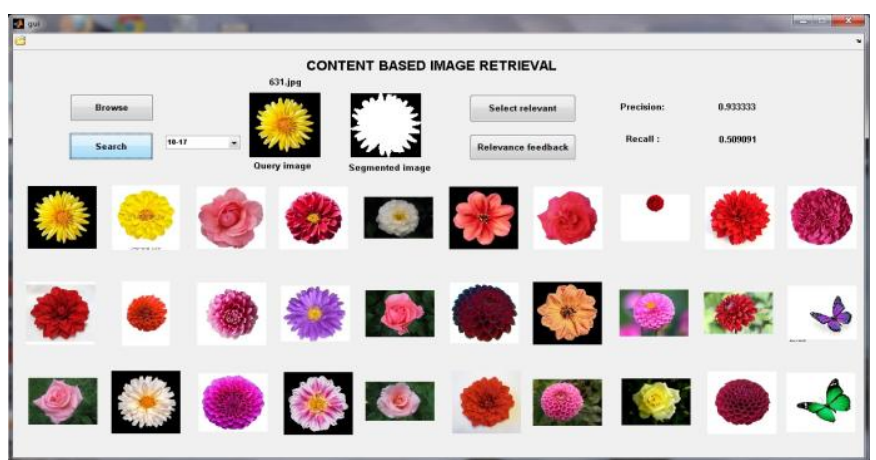

Fig.8. Results of flower as a query image.

4.1.3 Results using moments between order 3-17:

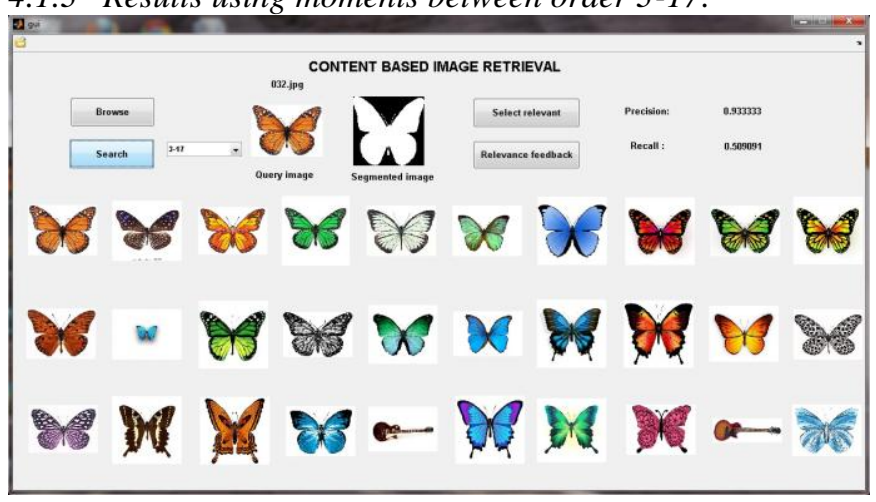

Fig.9. Results of butterfly as a query image. 


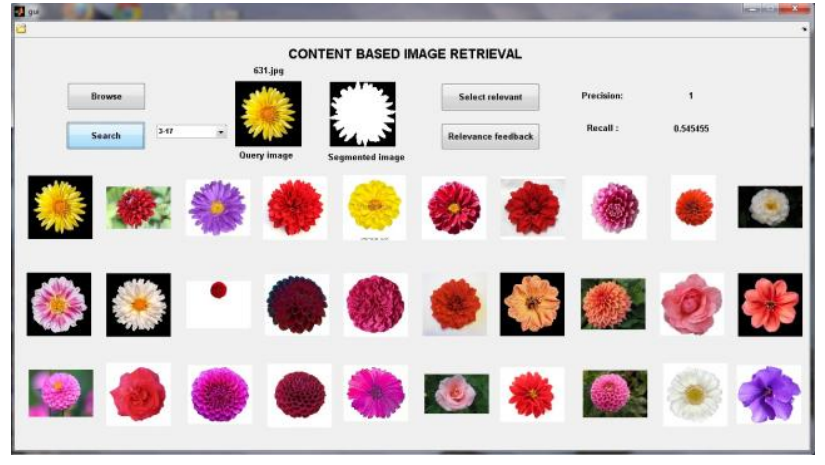

Fig.10. Results of flower as a query image.

\subsection{Statistical Results:}

The statistical results using precision and recall are as shown in Table II. Two sets of features each containing 32 Zernike moments and one more set containing 61 Zernike moments with different orders are extracted from the images. The experimental results shows that moments of order 3-10 gives better precision and recall than 10-17 and 3-17 set of moments order. The analysis of all the 3 sets with various other categories is also presented in Table II. The proposed system is being implemented with the latest and efficient techniques that can better handle inaccuracies of the geometric moments.

The analysis shows that higher order moment upto 10 are better shape descriptors. The increase in the moments order decreases the computational speed. The higher order moments also results in increase in complexity and approximation error. The higher order Zernike moments are the accumulations of the lower order computed Zernike moments, when the order of Zernike moments goes higher the accumulated errors would quickly get out of control and the use of higher order Zernike moments would be severely handicapped. But, it is possible to overcome such errors. If the approximation error is controlled then the higher order Zernike moments can give better results. Region based retrieval systems performance is greatly affected by the segmentation process. Development of an improved image segmentation algorithm could be one of the future scopes.

Table II. Statistical Results

\begin{tabular}{|l|l|l|l|l|l|l|}
\hline \multirow{2}{*}{ Category } & \multicolumn{2}{|c|}{$3-10$} & \multicolumn{2}{c|}{$10-17$} & \multicolumn{2}{c|}{$3-17$} \\
\cline { 2 - 7 } & Precision & Recall & Precision & Recall & Precision & Recall \\
\hline Butterfly & 0.8266 & 0.4509 & 0.5969 & 0.325 & 0.8 & 0.436 \\
& & & & 6 & & 3 \\
\hline Guitar & 0.8091 & 0.4413 & 0.6781 & 0.369 & 0.7703 & 0.420 \\
& & & & 9 & & 1 \\
\hline Teapot & 0.8678 & 0.4733 & 0.6896 & 0.376 & 0.8254 & 0.450 \\
& & & & 1 & & 2 \\
\hline Dinosaurs & 0.8557 & 0.4667 & 0.4569 & 0.251 & 0.7981 & $\begin{array}{l}0.435 \\
3\end{array}$ \\
\hline Flowers & 0.9315 & 0.5080 & 0.7684 & 0.419 & 0.9284 & 0.506 \\
& & & & 1 & & 4 \\
\hline Dolls & 0.7939 & 0.4330 & 0.3812 & 0.207 & 0.7082 & 0.386 \\
& & & & 9 & & 1 \\
\hline
\end{tabular}

\section{CONCLUSION AND FUTURE SCOPE}

An efficient three stage CBIR technique using shape as the feature with appreciating precision and recall is implemented. A novel analysis of different orders of Zernike moments is also presented. The experimental analysis proves that a Zernike moments upto the highest order of 10 gives better results than moments of order upto 17 . The future scope can be to merge the system with other features like colour or texture or with any learning system which can classify images on the basis of objects.

\section{REFERENCES}

[1] Dipti Jadhav, Gargi Phadke, Satish Devane, "Novel Weight Allocation technique for Image Retrieval using Higher Order Color Moments and CCM Texture Features", ICALIP, IEEE 2012,pg 129-133, Shanghai,China.

[2] AbhinavDeshpande, S K Tadse, “ Design Approach for Content - Based Image Retrieval using Gabor- Zernike features", Vol.4, No.04, April 2012.

[3] Basavaraj S. Anami, Suvarna S Nandyal, A. Govardhan, "Suitability of Shape and Texture Features in Retrieval Medicinal Plant Images in Indian Context", Indian Journal of Machine Learning and Computing, Vol 2, No December 2012.

[4] Dipti Jadhav, Gargi Phadke, Satish Devane, "Colour and Texture Feature Based Hybrid Appraoch for Image Retrieval", Advances in Computer Science, Eng. And Applications., AISC 166, pg 101-111, Springer-Verlag Berlin, Heidelberg 2012

[5] Hongli Tian, Huiqiang Yan, Hongdong Zhao, "A Fast and Accurate Approach to the Computation of Zernike Moments", Applied Informatics and Communications, Communications in Computer and Information Science, Vol 228, 2011, pg. 46-53.

[6] Manimala Singh and K Hemachandran, "Content Based Image Retrieval Using Color and Texture", Signal and Image Processing : An International Journal(SIPIJ), Vol 3, No 1 ,Febraury 2012.

[7] Shriram K V, P.L.K Priyadarsini, Subashri V., “ CBIR An Analysis and Suggestion for Improvement." An International Journal of Computer Applications(09758887, Volume 42, No. 14, March 2012).

[8] Dengsheng Zhang, Guojun Lu, "Review of Shape Representation and Description Techniques", Pattern Recognition 37 2004, 1-19, Elsevier.

[9] Tohid Sheghi, "Features Composition For Proficient and Real Time Retrieval In CBIR System", Journal of Asian Scientific Research, 2013, 3(1); 68-74.

[10] Dengsheng Zhang, Guojun Lu, "A Comparative Study of Three Region Shape Descriptors", DICTA2002: Digital Image Computing Techniques and Applications, 21-22 Jan-02.

[11] Ye Bin and PengJia-xiong, "Improvement and Invariance Analysis of Zernike Moments using as a Region based Shape Descriptor", SIBGRAPI '02 Proceedings of the 15th Brazilian Symposium on Computer Graphics and Image Processing, 2002.

[12] Amir Tahmasbin, Fatemeh Saki, Shahriar B.Shokouhi, "Classification of benign and malignant masses based on 
Zernike moments", Comput Bio Med. 2011, Aug; 41(8):726-35 doi:10.1016/j.compbiomed.2011.06.009. Epub $2011 \mathrm{Jul} 1$.

[13] Xinmeng Liao, "Image Analysis by Moments. Simon Xinmeng Liao 1993, The University of Manitoba, Winnipeg, Manitoba, Canada.

[14] B. Ramamurthy, K.R.Chandran, "Content based Image Retrieval for Medical ImagesUsing Canny Edge Detection" Algorithm International Journal of Computer Applications (0975 - 8887) Volume 17- No.6, March 2011
[15] Ch. Srinivasa Rao , S.Srinivas Kumar and B.Chandra Mohan, "Content Based Image Retrieval using Exact Legendre Moments and Support Vector Machine", The International Journal of Multimedia \& its Applications (IJMA), Vol.2, No.2, May2010.

[16] Tsong-Wuu Lin, Yun-Feng Chou, "A Comparative Study of Zernike Moments for Image Retrieval", 16th IPPR Conference on Computer Vision, Graphics and Image Processing (CVGIP 2003)2003. 